\title{
BMJ Open Study protocol to develop a core outcome set for thyroid dysfunction to bridge the unmet needs of patient- centred care
}

\author{
Cheow Peng Ooi (D) , ${ }^{1,2}$ Abdul Hanif Khan Yusof Khan (D) , ${ }^{3}$ Rosliza Abdul Manaf, ${ }^{4}$ \\ Norlaila Mustafa, ${ }^{5}$ Norlela Sukor, ${ }^{5}$ Paula R Williamson, ${ }^{6}$ Nor Azmi Kamaruddin ${ }^{5}$
}

To cite: 0 oi $C P$, Yusof Khan AHK, Abdul Manaf R, et al. Study protocol to develop a core outcome set for thyroid dysfunction to bridge the unmet needs of patient-centred care. BMJ Open 2021;11:e050231. doi:10.1136/ bmjopen-2021-050231

- Prepublication history and additional supplemental material for this paper are available online. To view these files, please visit the journal online. To view these files, please visit the journal online (http://dx. doi org/10.1136/bmjopen-2021050231).

Received 14 February 2021 Accepted 13 July 2021

Check for updates

(C) Author(s) (or their employer(s)) 2021. Re-use permitted under CC BY-NC. No commercial re-use. See rights and permissions. Published by BMJ.

For numbered affiliations see end of article.

Correspondence to

Dr Cheow Peng 0oi;

cheowpeng@upm.edu.my
Abstract

Introduction Thyroid dysfunctions (TD) are common medical conditions affecting all global populations. Improved healthcare leading to increasing survival rates and delayed diagnosis rendered significant burden of the disease in the increasing number of patients with TD with comorbid illnesses. Therefore, reducing the burden of TD and improving the quality of care are crucial. Existing poor-quality data that guide evidence-based decisions only provide a fragmented picture of clinical care. The different outcomes across studies assessing the effectiveness of treatments impede our ability to synthesise results for determining the most efficient treatments. This project aims to produce a core outcome set (COS), which embeds the multiple complex dimensions of routine clinical care for the effectiveness studies and clinical care of adult patients with TD.

Methods and analysis This mixed-method project has two phases. In phase 1, we will identify a list of patientreported and clinical outcomes through qualitative research and systematic reviews. In phase 2, we will categorise the identified outcomes using the Core Outcome Measures in Effectiveness Trials taxonomy of core domains and the International Classification of Functioning, Disability and Health. We will develop questionnaires from the list of outcomes identified from each domain for the two-round online Delphi exercise, aiming to reach a consensus on the COS. The Delphi process will include patients, carers, researchers and healthcare participants. We will hold an online consensus meeting involving representatives of all key stakeholders to establish the final COS.

Ethics and dissemination The study has been reviewed and approved by the Ethics Committee for Research Involving Human Subjects, Universiti Putra Malaysia and the Research Ethics Committee, National University of Malaysia. This proposed COS in TD will improve the value of data, facilitate high-quality evidence synthesis and evidence-based decision-making. Furthermore, we will present the results to participants, in peer-reviewed academic journals and conferences.

Registration details Core Outcome Measures in Effectiveness Trials (COMET) Initiative database registration: http://www.comet-initiative.org/studies/ details/1371

\section{Strengths and limitations of this study}

- This protocol is guided by the Core Outcome SetSTAndards for Development recommendations of the Core Outcome Measures in Effectiveness Trials initiative, a robust methodology, to develop a set of globally relevant core outcomes to evaluate thyroid dysfunction in adults.

- This core outcome set for thyroid dysfunction will reduce both heterogeneity and outcome reporting bias between studies, allowing collation of results of primary clinical research to improve evidence synthesis in the field.

- An international study steering committee will supervise this study, and multidisciplinary stakeholders in thyroid care that include clinicians, epidemiologists, core outcome research experts and patients will be engaged.

- Systematic reviews and qualitative research approach will identify the long list of outcomes for the Delphi consensus.

- Further study will be required to identify outcome measurement instruments to assess the outcome selected.

\section{INTRODUCTION}

Thyroid dysfunction (TD) affects all populations globally and encompasses the entire spectrum of common disorders, ranging from under secretion to over secretion of thyroid hormones, triiodothyronine (T3) and thyroxine (T4). ${ }^{1}$ The global incidence and prevalence of TD are difficult to compare because of differences in data on diagnostic thresholds, assay sensitivities, population selection and fluxes in iodine nutrition, highlighting geographical differences and the effect of environmental factors such as iodine supplementation. Strategies for early detection and intervention, the high-quality of healthcare services, progress in treatment options and emphasis on safe medication have increased survival rates and the ageing 
population in developed countries. However, patients with TD often have comorbid illnesses besides the longterm and short-term disease burden. ${ }^{2}$ Undiagnosed persons with TD are common in developing countries, and the precise burden is not known. In Malaysia, 7 out of 10 persons with TD did not know they have TD. ${ }^{3}$ There were no detailed epidemiological surveys in many other developing countries. Undiagnosed TD may put patients at risk for serious complications, for example, cardiovascular diseases, osteoporosis and infertility. The global burden of multimorbidity and disability is estimated to be $84 \%$ of the burden in 2008 and projected to increase to $89 \%$ by $2030 .{ }^{4}$ Despite the complex management of adult patients with $\mathrm{TD}$, the devastating health consequences that affect all populations worldwide are potentially preventable. $^{1}$

Thyroid hormone derangements across both ends of the TD spectrum have negative impacts on the heart. ${ }^{5}$ Delayed diagnosis or suboptimal management of hyperthyroidism results in a prolonged period of increased thyroid hormones. The resulting complications, such as atrial fibrillation, thyrotoxic cardiomyopathy, cardiac failure and cardioembolic stroke, may increase mortality or disability. While atrial fibrillation may be transient, disability from a cardioembolic stroke may be permanent.

The long-term disease burden of subclinical hyperthyroidism includes the progression to overt hyperthyroidism, increased risk of atrial fibrillation, osteoporosis and worsened prognosis in an acute ischaemic stroke. ${ }^{67} \mathrm{In}$ contrast, asymptomatic subclinical hypothyroidism with thyroid-stimulating hormone (TSH) levels $<10 \mathrm{mIU} / \mathrm{L}$ may have no detrimental health effects in older adults, and treatment may induce iatrogenic subclinical or overt hyperthyroidism and the associated complications. ${ }^{8}$ Thus, reducing the long-term and short-term burden of TD is critically essential.

Despite the strive for clinical research in TD to improve the quality of patient care, formidable challenges remain. These challenges range from inadequate clinical assessment and the overuse of laboratory thyroid function testing to conflicting findings of interventions in clinical research, inadequate understanding of patient experiences and the impact of TD in the real world. ${ }^{9}$ Besides the discrepancies between the results of TSH, T3 and $\mathrm{T} 4$ assays, clinical features and the biochemical profiles may present discordant information, hampering the diagnostic and decision-making processes. ${ }^{10}$ Although a randomised clinical trial is the gold standard in evaluating interventions, it often cannot capture the richness and complexity of the multidimensional aspects of clinical care at the patients' level. ${ }^{11}$ Taken together, these issues emphasise the need for a comprehensive and integrative evaluation of all the biopsychosocial components of patient care, including health equity, to facilitate the translation of new interventions or care components optimally to benefit both patients and all stakeholders.

Recent clinical guidelines and systematic reviews have highlighted the lack of high-quality evidence regarding
TD management. ${ }^{12-20}$ Seven systematic reviews evaluating treatment of TD in adults were identified in the Cochrane library. ${ }^{14-20}$ These systematic reviews have highlighted the lack of high-quality evidence regarding the management of TDs. Although one review could conclude the effects of antithyroid treatment on Graves' disease, the results needed to be interpreted cautiously. ${ }^{14}$ Part of the reason for this is the large number of varied outcomes and clinical indices that are measured within the trials to compare the effectiveness of the management options. ${ }^{21}$ This heterogeneity of outcome measures makes it difficult to compare or combine different study results, confusing patients and clinicians when deciding the best treatment options. Currently, there is no consensus on which outcomes should be measured in clinical research or clinical practice in the field of TD.

A core outcome set (COS) is an agreed standardised collection of outcomes that should be measured and reported by all research or trials for a specific clinical area. ${ }^{22}$ Using a COS in future trials of interventions in adult patients with TD serves to increase the value of the data and promote the development of relevant, highquality evidence for decision-making. ${ }^{23}$ Minimising the inconsistency of outcomes reported by trials will enable an increase in the number of patients and statistical power for meaningful meta-analysis. ${ }^{22}$ The increased value of research data will facilitate the translation of interventions to the clinical arena. Increasing uptake of the highquality, relevant evidence in the targeted population will reduce research waste. Finally, a multidimensional COS, reflective of all health service users, including patients, is promoted by the WHO, the Cochrane reviews of effects of healthcare interventions and is adopted by multiple disciplines in medicine. ${ }^{24-29}$

\section{AIMS AND OBJECTIVES}

We aimed to produce a COS, which embeds the complex multidimensions of routine clinical care and effectiveness studies of adult patients with TD that are relevant to all stakeholders of TD internationally.

Objectives of this study are as follows:

1. To identify all potential outcomes for adults with TD in clinical trials, routine clinical care, patients' registries, the existing literature and from qualitative research on outcomes that matter to patients with TD.

2. To achieve consensus on a COS for adults with TD using the Delphi and nominal group techniques to develop the final COS set.

\section{SCOPE}

\section{Inclusion criteria:}

This COS will be developed for clinical studies reflective of issues in real-life practice evaluating diagnosis, treatment and follow-up of the range of TD from hyperthyroidism to hypothyroidism in adults. The whole range of TD is addressed because hormonal fluctuations from one 
end of the spectrum to another are common in thyroid diseases. For example, Graves' disease is a common cause of thyrotoxicosis. Remission (normal thyroid hormone level) or hypothyroidism may occur with or without treatment. Likewise, overtreatment for hypothyroidism may cause hyperthyroidism. Hence, a COS that can capture the entire spectrum of thyroid hormonal fluctuations is important for comprehensive management of thyroid diseases.

The other inclusion criteria are:

1. Adult patients with TD over 18 years old, including man and woman as well as older adults with TD.

2. Patients who have undergone surgical removal of the thyroid gland and/or radioactive iodine ablation requiring continual active medical surveillance and management.

3. Patients who have history of hospital of hospital admissions for TD, including thyroid emergencies, for example, thyroid storm.

\section{Exclusion criteria}

The exclusion criteria are:

1. Patients undergoing active treatment for thyroid cancer.

2. Patients undergoing active treatment for dysthyroid eye disease.

3. Patients who are pregnant.

4. Patients undergoing surgical interventions to treat TDs.

5. Patients undergoing radioactive iodine ablation procedures to treat thyroid diseases.

\section{Stakeholders}

1. Patients with TD and carers/families of patients with TD.

2. Healthcare professionals include endocrinologists, physicians who care for patients with TD, surgeons who provide pre and postsurgical care for patients with $\mathrm{TD}$, other allied healthcare professionals involved in the care of TD and policymakers.

3. Researchers of TD.

\section{Intervention}

Any intervention used in the treatment of TD.

\section{METHODS AND ANALYSIS \\ Study design}

This study will adhere to the recommended guidelines in the Core Outcome Measures in Effectiveness Trials (COMET) handbook and COS-Standards for Development (COS-STAD). ${ }^{30} 31$ The completeness of this protocol was assessed using the Core Outcome Set-STAndardised Protocol Items (COS-STAP) checklist. ${ }^{32}$ The completed COS-STAD and COS-STAP checklists can be found in online supplemental S1 and S2, respectively.

We used a mixed-method design involving both qualitative and quantitative methodologies to achieve consensus on the COS. The steps are summarised in figure 1.

\section{Study steering committee}

The multidisciplinary study steering committee (SSC) comprising senior consultant endocrinologists, expert COS researcher, epidemiologist, physicians and patient

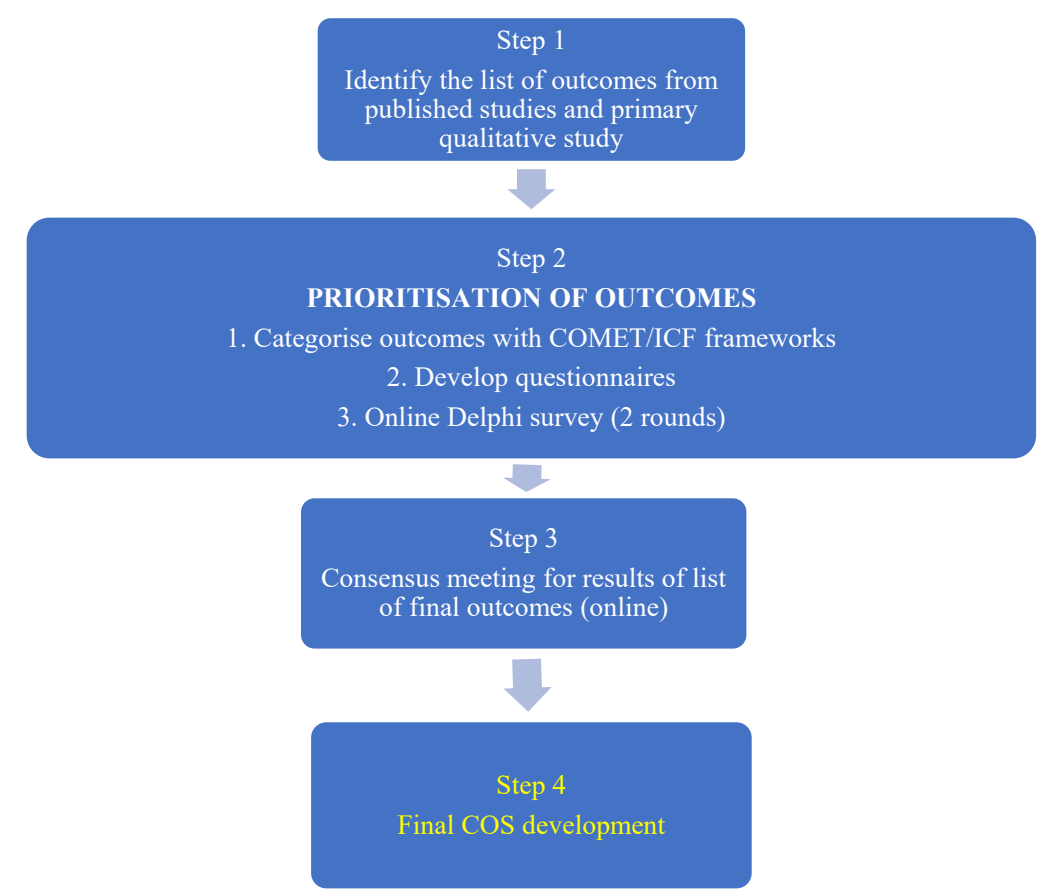

Figure 1 Steps in the development of TD-COS. COMET, Core Outcome Measures in Effectiveness Trials; COS, core outcome set; ICF, International Classification of Functioning; TD, thyroid dysfunction. 
researchers will oversee the COS study; provide feedback on the study protocol, list of outcomes; disseminate the online Delphi survey; contribute to the final consensus meeting and dissemination of the final COS. We are engaging patients and other critical stakeholders of thyroid care in the SSC as they offer unique and valuable experiences and perspectives aligning with real-world needs and concerns. ${ }^{33} 34$ Their engagements as partners in research have demonstrated improvements in the relevance of research questions, design, implementation and dissemination of patient-centred research. ${ }^{35}$ Besides increasing the transparency of research activities, the findings that target the concerns and care needs of the patients with TD will also facilitate the translation of evidence to the clinical practice arena.

\section{Step 1: generating the outcomes list}

We will establish a patient care pathway through the consensus from clinical content experts to provide a conceptual framework to identify potential outcomes. We will organise online meetings to consult clinical content experts on the scope of the patient care pathway for patients with TD and the development process. The following sources will generate the list of outcomes for an online Delphi survey:

1. Systematic reviews.

We will search EMBASE, Medline and Cochrane Library to identify studies on any interventions for TD. In addition, we will include studies most likely to influence clinical practice according to the Oxford Centre for Evidence-Based Medicine's guidelines on study hierarchy. These clinical studies include any clinical studies, clinical trials, prospective and retrospective cohort studies.

2. Outcomes from other sources

We will identify outcomes from measures used in routine clinical care, patients' registries, clinical trial registries, clinical practice guidelines on thyroid disorders, hyperthyroidism and hypothyroidism.

\section{Systematic literature review}

We will explore the literature from the past 10 years, so that the outcomes extracted reflect the evidence for the current treatment of TD in adults. Any intervention used for the treatment of TD will be included. Clinical outcomes and outcome measurement tools will be those relevant for assessing patient recovery and well-being, short-term outcomes (eg, adverse events and complications of surgical and non-surgical care) and long-term outcomes (eg, quality of life). Conference abstracts and non-English-language publications will be excluded.

Two reviewers will independently assess the abstracts of studies resulting from our literature search. Then, another two reviewers will independently assess full-text copies of studies meeting the inclusion criteria. Finally, the members of the SSC will discuss and agree on the included studies for outcome extraction.

\section{Data analysis and presentation}

All outcomes reported in the eligible studies will be extracted, tabulated with their definition and measurement method(s) and then categorised into domains. To ensure the comprehensiveness of COS, we will assign outcomes terms according to the COMET taxonomy of core domains and the International Classification of Functioning, Disability and Health for functional outcomes. ${ }^{36} 37$ The former has a taxonomy of 38 domains across five areas: death, physiological/clinical, life impact, resource use and adverse events. These categorisations will ensure adequate reporting of the results.

The members of the SSC will review the outcome list to ensure that the categorisation is appropriate; the outcome description is clear with no duplications. The SSC will also consider the number of outcomes and may request that outcomes be further condensed, combined or removed. For example, if the outcome is from a single or small number of studies or deemed irrelevant to TD interventions, it will be removed. Such an approach will ensure that the number of outcomes to be scored by participants of the online Delphi survey is manageable.

\section{Patient-reported outcomes and patients' perspectives}

The outcomes of patients with TD considered important in Malaysia will be identified from our qualitative research on 'What matters most to patients with TDs' ${ }^{38}$ Data from semistructured questionnaires were collected using both face-to-face in-depth interview technique and online survey. The participants included both genders, older population above 65 years old and the lower socioeconomic group from both urban and rural regions. Careful assessment will be made by the SSC team.

We will also search for existing patient-reported outcomes by evaluating tools and scales for TDs from publications. Finally, a meta-review will be carried to assess the outcomes reported in TD through the questionnaires, tools and scales used in TD research.

\section{Data extraction}

Data from the literature will be extracted independently by two reviewers, who will review the extracted data to assess consensus and ensure all outcomes are identified. With specific reference to outcomes, the following data will be extracted from each study: intervention(s) under investigation; each outcome reported; whether the outcome was defined; the definition used; the indicators/ tool(s) used to operationalise or measure the outcome; the time point or period of outcome measurement and how the outcome was reported. Disagreement will be resolved through discussion and third reviewer arbitration as required. In addition, we will contact study authors to identify any unclear/unavailable data.

\section{Data analysis and presentation}

We will enter the data into Microsoft Excel in order to aid tabulation and analysis. Outcomes will be grouped under domains following a review of the outcomes. The SSC will 
review the outcome domains and included outcomes to assess the suitability of the domain name and outcome grouping.

\section{Step 2: prioritisation of outcomes}

The goal of prioritising outcomes is to determine which outcomes the stakeholders think should be included in a COS for patients with TD. Measures from routine clinical care, patients' registries, international clinical practice guidelines on thyroid disorders, hyperthyroidism and hypothyroidism as well as the outcomes identified from the systematic literature reviews and qualitative interviews will create a long list of outcomes. ${ }^{31} 39$ These outcomes will be condensed and categorised using the COMET taxonomy of core domains and the International Classification of Functioning (ICF), Disability and Health for functional outcomes. ${ }^{36} 37$

The members of the SSC team will review and agree on the list of categorised outcomes. Since the outcomes measured in clinical effectiveness studies and clinical care must be responsive to all stakeholders' real-world needs and concerns, we will also engage, consult and seek input from the key stakeholders who will be the potential users of a COS for TD. ${ }^{33-35}$ This additional input on the preliminary outcome list will include other expert healthcare professionals caring for patients with TD, representatives from patient advocacy groups and policymaker. The final list of selected outcomes will be developed into questionnaires for the Delphi survey. Before entry to DelphiManager, we will seek the views of representatives of all the stakeholders' groups on the clarity of language, lay and medical terms used in the questionnaires. We will also engage patient research partners to facilitate the process.

\section{Delphi survey}

Although there are no strict recommendations for the number of participants (patients, carers, researchers and healthcare professionals) required in a Delphi study to gain consensus, having large and international participation from diverse stakeholders will increase the reliability of the group judgement. ${ }^{40}{ }^{41}$ We will invite all the stakeholder groups to take part in the consensus process using DelphiManager, a web-based system designed for Delphi studies. ${ }^{42}$ Besides minimal carbon footprint, this platform is also the best option for involving international experts, patients and the public, given the time and financial constraints. In addition, we will leverage on our networks in national and international professional societies for endocrinology and medicine as well as networks of COS researchers to connect to the potential stakeholders nationally and internationally.

The other advantage of the Delphi approach is the anonymous reviewing and scoring of outcomes in a manner that gives equal influence to all participants. Since there is no direct contact between participants, this technique avoids an individual participant being overtly influenced by the opinions of other participant and provides a mechanism for reconciling different opinions. ${ }^{43}$

\begin{tabular}{|c|c|c|}
\hline $\begin{array}{l}\text { Classification } \\
\text { of a consensus }\end{array}$ & Description & Definition \\
\hline In & $\begin{array}{l}\text { The consensus } \\
\text { that the outcome } \\
\text { should be } \\
\text { included in the } \\
\text { core outcome set. }\end{array}$ & $\begin{array}{l}\geq 70 \% \text { of participants } \\
\text { scoring as } 7-9 \text { and } \\
<15 \% \text { of participants } \\
\text { scoring as } 1-3 \text { in both } \\
\text { stakeholder groups. }\end{array}$ \\
\hline Out & $\begin{array}{l}\text { The consensus } \\
\text { that the outcome } \\
\text { should not be } \\
\text { included in the } \\
\text { core outcome set. }\end{array}$ & $\begin{array}{l}\leq 50 \% \text { of participants } \\
\text { scoring } 7-9 \text { in both } \\
\text { stakeholder groups. }\end{array}$ \\
\hline No consensus & $\begin{array}{l}\text { Uncertainty about } \\
\text { the importance of } \\
\text { the outcome. }\end{array}$ & Anything else. \\
\hline
\end{tabular}

Questionnaires developed from the selected outcomes will be sent using DelphiManager. ${ }^{42}$ Consent will be picked up as implicit for Delphi participants, who will be required to register via the website to submit the completed questionnaires.

\section{Delphi round 1}

The selected participants will reflect a broad range of clinical experiences and geographical expertise, with representations from both the developed and developing countries. In round 1 of the Delphi survey, demographic data on the participants (eg, stakeholder group category, professional background and experience with clinical research relevant to TD) will be collected. The outcome order will be randomly assigned to mitigate the influence of the display order on scoring. Participants will rank each outcome on a Likert scale of 1-9, developed from the Grading of Recommendations Assessment, Development and Evaluation (GRADE) working group definitions (table 1) and the recommendations of the COMET handbook. ${ }^{31}{ }^{44}$ The focus will be on rating the most important outcomes for inclusion as high and excluding outcomes felt to be of lesser importance. Regardless of the score, all outcomes will be carried forward to the second round.

Through free-text entry, participants will also have the option to clarify compelling arguments for and against including the outcomes or suggest additional outcomes not included in the round 1 questionnaire. These suggestions will be reviewed and considered for inclusion into round 2 of the Delphi if the TD-COS study team is of the consensus that the outcome does not reflect or is not similar to another outcome already listed.

After analysing responses from round 1, we will collate a feedback report. The analyses of the questionnaire responses and scores from each stakeholder group will be presented separately. The SSC team will review and evaluate responses for substantial arguments. Additional suggestions will be reviewed for uncaptured outcomes from round 1 . We will not invite panellists who do not complete round 1 to participate in round 2 . 


\section{Delphi round 2}

In Delphi round 2, we will summarise the responses for each stakeholder group for each outcome. These summaries will be displayed graphically as the percentage of each stakeholder group who has given each score. The participants will view the responses from the previous round of the Delphi exercise and can decide to keep or change their initial rating. New outcomes developed from free-text responses in round 1 will be presented in round 2 alongside the verbatim text of the outcome. The participants will be asked to score these new outcomes. These activities will lead to the group converging on a consensus opinion through the course of these two rounds. Responses from round 2 will be analysed, and the scoring will be similarly based on the GRADE recommendations used for round 1 (table 1 ).

\section{Data collection and confidentiality}

All data will be handled confidentially and in accordance with General Data Protection Regulation, the Data Protection Act 2018 of the UK, Personal Data Protection Act of Malaysia and data protection legislation of the respective countries of the participants. Access to personally identifiable data will be strictly limited to identifying information (name and email) obtained during registration and delegated members of the research team. This information, used only for direct feedback and reminder emails, will be stored separately from the answers given in the questionnaire. Furthermore, data from answers to questionnaires collected from the e-Delphi survey will be encrypted and securely stored anonymously by the DelphiManager server, hosted by the University of Liverpool, UK. ${ }^{42}$ On completion of this research project, the data will be archived and stored for a period of 5 years.

\section{Stage 3: consensus meeting}

We will organise an online meeting comprising the SSC and selected panel members representing all stakeholders: endocrinologists, researchers, representatives of patient advocacy groups, patients with TD, clinicians, other healthcare professionals and policymaker. These keystakeholders not only have deep insight into the realworld needs and concerns of thyroid care but are also potential users of a COS for TD. ${ }^{33-35}$ All outcomes from round 2 of the e-Delphi survey will be presented. Both the aggregate score for each outcome and the individual score for each stakeholder group will be displayed to ensure that any important differences are highlighted and discussed. Participants will then anonymously vote for each outcome for inclusion and exclusion in the final COS using a format similar to that of the Delphi survey. We will categorise the results of the consensus based on the definitions in table 1 . Attendees will also suggest outcomes that warrant further discussion, including those that have reached no consensus. If a final COS is not agreed on at the end of the first online consensus meeting, subsequent online meetings will be considered.
Stage 4: final COS development

This study aims to achieve a pragmatic COS applicable and feasible for all future clinical studies, including clinical trials, that evaluate TD treatment and clinical care in adults. Even though there is no recommended maximum number of outcomes that should be included in a COS, the number needs to be feasible for practice. Since the outcomes for TD endeavour to achieve a balance between standardisation and comprehensiveness of outcome reporting, outcomes representing all core outcome domains, mortality/survival; clinical/physiological; life impact/functioning; resource use and adverse events, will be included. ${ }^{3645}$ Therefore, there will be no limit on the final number of outcomes. Stratified analyses of the Delphi results will be performed to test for country bias. The final COS will be categorised according to the COMET/ICF taxonomy. ${ }^{36}{ }^{37}$ We will also annotate the outcomes according to a recently published outcome taxonomy to maximise future data harmonisation.

\section{Patient and public involvement}

Patients and public have been invited to provide input in the inception and design of the different stages of this project. We will carefully assess the burden of participation in the different stages of the study.

\section{ETHICS AND DISSEMINATION}

This project in developing the protocol has received ethical approval from both Universiti Putra Malaysia (JKEUPM-2019-349) and National University of Malaysia Medical Centre (UKM PPI/111/8/JEP-2019-758). Informed written consent will be sought from participants prior to the interviews. Consent will be taken as implicit for Delphi participants, who will be required to register via the website to submit the completed questionnaires. This COS development project is registered on COMET, an international public COS database (http:// www.comet-initiative.org/studies/details/1371).

By observing the COMET, COS-STAD and COS-STAP recommendations, this protocol represents a robust method for COS development. In each phase along the research process, there is the active participation of patients, international healthcare professionals and academics. In addition, a patient and clinical advisory group will oversee the project to ensure that the only outcomes important to all key stakeholders are represented in the final COS.

On the agreement to the final COS, we plan to develop a core outcome measurement instrument set. The systematic reviews in this protocol will have identified existing measurement instruments. A complementary comprehensive literature search, quality assessment of the measurement instruments and consensus of key stakeholders will guide the selection of candidate measures. We will base the methodology on the latest recommendations to select measures that have the highest validity, reliability and responsiveness in the adult TD population. ${ }^{46} 47$ 
Engaging a wide range of international participants from all stakeholder groups will facilitate future dissemination, uptake and use of the COS in this area of healthcare. The results will be presented to all the participants, in peer-reviewed academic journals and at (international) conferences. The successful implementation of COS in other healthcare fields has improved quality and relevance of research with increased reliability of evidence synthesis on which robust clinical guidelines can be based. ${ }^{24} 2648$ Improve shared decision-making enhanced the treatment process when outcomes in TD are relevant to both clinicians and patients. Furthermore, a COS for TD will better equip healthcare providers on how best to prioritise funding for interventions that reflect the needs and priorities of patients. Finally, we anticipate that a COS for adults with TD will do the same in improving the quality of clinical trials, clinical decision-making, patient care and policymaking.

\section{Author affiliations}

${ }^{1}$ Endocrine Unit, Department of Medicine, Universiti Putra Malaysia Faculty of Medicine and Health Sciences, Serdang, Selangor, Malaysia

${ }^{2}$ Endocrine Unit, Department of Medicine, Pusat Perubatan Universiti Kebangsaan Malaysia, Cheras, Malaysia

${ }^{3}$ Department of Neurology, Universiti Putra Malaysia Faculty of Medicine and Health Sciences, Serdang, Selangor, Malaysia

${ }^{4}$ Community Health, University Putra Malaysia, Serdang, Selangor, Malaysia

${ }^{5}$ Department of Medicine, Pusat Perubatan Universiti Kebangsaan Malaysia, Cheras, Kuala Lumpur, Malaysia

${ }^{6}$ Biostatistics, The University of Liverpool, Liverpool, UK

Acknowledgements The authors would like to acknowledge and sincerely thank Mrs Sharifah Binti Mohd Nor, our patient researcher, and members of the 'Tiroid' support group, for providing critical input in the study conception, study design and help identify the relevant target areas for developing this research protocol.

Contributors $\mathrm{CPO}$ initiated the project and drafted this protocol. This protocol was refined by RAM, AHKYK, NAK, NM, NS, PRW. All authors contributed to the design, manuscript, read and approved the final manuscript. NAK is the head of the study steering committee, PRW is the leading COS research expert, and all authors are members of the study steering committee.

Funding The work was supported by Universiti Putra Malaysia Research Grant (UPM800/3/3/1/GPIPM/2018/9641400).

Competing interests None declared.

Patient consent for publication Not required.

Provenance and peer review Not commissioned; externally peer reviewed.

Supplemental material This content has been supplied by the author(s). It has not been vetted by BMJ Publishing Group Limited (BMJ) and may not have been peer-reviewed. Any opinions or recommendations discussed are solely those of the author(s) and are not endorsed by BMJ. BMJ disclaims all liability and responsibility arising from any reliance placed on the content. Where the content includes any translated material, BMJ does not warrant the accuracy and reliability of the translations (including but not limited to local regulations, clinical guidelines, terminology, drug names and drug dosages), and is not responsible for any error and/or omissions arising from translation and adaptation or otherwise.

Open access This is an open access article distributed in accordance with the Creative Commons Attribution Non Commercial (CC BY-NC 4.0) license, which permits others to distribute, remix, adapt, build upon this work non-commercially, and license their derivative works on different terms, provided the original work is properly cited, appropriate credit is given, any changes made indicated, and the use is non-commercial. See: http://creativecommons.org/licenses/by-nc/4.0/.

\section{ORCID iDs}

Cheow Peng 0oi http://orcid.org/0000-0003-4431-0521

Abdul Hanif Khan Yusof Khan http://orcid.org/0000-0002-8975-2174

\section{REFERENCES}

1 Taylor PN, Albrecht D, Scholz A, et al. Global epidemiology of hyperthyroidism and hypothyroidism. Nat Rev Endocrinol 2018;14:301-16.

2 Biondi B, Kahaly GJ, Robertson RP. Thyroid dysfunction and diabetes mellitus: two closely associated disorders. Endocr Rev 2019;40:789-824.

3 Thyroid Guidelines Development Group. Clinical Practice Guidelines: Management of Thyroid Disorders. In: M M, ed. Clinical practice guidelines. Malaysia: Malaysian Endocrine And Metabolic Society (MEMS), 2019.

4 James SL, Abate D, Abate KH, et al. Global, regional, and national incidence, prevalence, and years lived with disability for 354 diseases and injuries for 195 countries and territories, 1990-2017: a systematic analysis for the global burden of disease study 2017 . Lancet 2018;392:1789-858.

5 Razvi S, Jabbar A, Pingitore A, et al. Thyroid hormones and cardiovascular function and diseases. J Am Coll Cardiol 2018;71:1781-96.

6 Woeber KA. An overview of endogenous subclinical hyperthyroidism and cardiac disease. Endocr Pract 2016;22:1449-52.

7 Yang R, Yao L, Fang Y, et al. The relationship between subclinica thyroid dysfunction and the risk of fracture or low bone mineral density: a systematic review and meta-analysis of cohort studies. $J$ Bone Miner Metab 2018;36:209-20.

8 Manolis AA, Manolis TA, Melita $\mathrm{H}$, et al. Subclinical thyroid dysfunction and cardiovascular consequences: an alarming wake-up call? Trends Cardiovasc Med 2020;30:57-69.

9 Todd $\mathrm{CH}$. Management of thyroid disorders in primary care: challenges and controversies. Postgrad Med J 2009;85:655-9.

10 Haddad RA, Giacherio D, Barkan AL. Interpretation of common endocrine laboratory tests: technical pitfalls, their mechanisms and practical considerations. Clin Diabetes Endocrinol 2019;5:12.

11 Heneghan C, Goldacre B, Mahtani KR. Why clinical trial outcomes fail to translate into benefits for patients. Trials 2017;18:122.

12 Jonklaas J, Bianco AC, Bauer AJ, et al. Guidelines for the treatment of hypothyroidism: prepared by the American thyroid association Task force on thyroid hormone replacement. Thyroid 2014;24:1670-751

13 Ross DS, Burch HB, Cooper DS. American thyroid association guidelines for diagnosis and management of hyperthyroidism and other causes of thyrotoxicosis. Thyroid 2016 2016;26:1343-421.

14 Abraham P, Avenell A, McGeoch SC, et al. Antithyroid drug regimen for treating Graves' hyperthyroidism. Cochrane Database Syst Rev 2010;70.

15 Ke L-qiu, Hu Y, Yang K, et al. Chinese herbal medicines for hypothyroidism. Cochrane Database Syst Rev 2015:CD008779.

16 Liu ZW, Masterson L, Fish B, et al. Thyroid surgery for Graves' disease and Graves' ophthalmopathy. Cochrane Database Syst Rev 2015;99.

17 Ma C, Xie J, Wang H, et al. Radioiodine therapy versus antithyroid medications for Graves' disease. Cochrane Database Syst Rev 2016;2:CD010094.

18 van Zuuren EJ. Selenium supplementation for Hashimoto's thyroiditis. Cochrane Database of Systematic Reviews 2013;6.

19 Villar $\mathrm{H}$. Thyroid hormone replacement for subclinical hypothyroidism. Cochrane Database of Systematic Reviews 2008;1.

20 Zen XX, Yuan Y, Liu Y, et al. Chinese herbal medicines for hyperthyroidism. Cochrane Database Syst Rev 2007;2:CD005450.

21 Sautenet B, Contentin L, Bigot A, et al. Strong heterogeneity of outcome reporting in systematic reviews. J Clin Epidemiol 2016;75:93-9.

22 Clarke M. Standardising outcomes for clinical trials and systematic reviews. Trials 2007;8:39.

23 Tunis SR, Clarke M, Gorst SL, et al. Improving the relevance and consistency of outcomes in comparative effectiveness research. $J$ Comp Eff Res 2016;5:193-205.

24 Khan K. The CROWN Initiative: Journal editors invite researchers to develop core outcomes in women's health. Best Pract Res Clin Obstet Gynaecol 2019;57:e1-4.

25 Maxwell LJ, Beaton DE, Shea BJ. Core domain set selection according to OMERACT filter 2.1: the OMERACT methodology. $J$ Rheumatol 2019

26 Prinsen CAC, Spuls PI, Kottner J, et al. Navigating the landscape of core outcome set development in dermatology. J Am Acad Dermatol 2019;81:297-305.

27 Rosenbaum SE, Glenton C, Oxman AD. Summary-of-findings tables in Cochrane reviews improved understanding and rapid retrieval of key information. J Clin Epidemiol 2010;63:620-6. 
28 Dodd S, Harman N, Taske N, et al. Core outcome sets through the healthcare ecosystem: the case of type 2 diabetes mellitus. Trials 2020;21:570.

29 WHO. WHO Handbook for Guideline Development. Geneva: World Health Organisation, 2014.

30 Kirkham JJ, Davis K, Altman DG, et al. Core outcome Set-STAndards for development: the COS-STAD recommendations. PLoS Med 2017;14:e1002447.

31 Williamson PR, Altman DG, Bagley H, et al. The comet Handbook: version 1.0. Trials 2017;18:280.

32 Kirkham JJ, Gorst S, Altman DG, et al. Core outcome SetSTAndardised protocol items: the COS-STAP statement. Trials 2019;20:116.

33 Concannon TW, Fuster M, Saunders T, et al. A systematic review of stakeholder engagement in comparative effectiveness and patient-centered outcomes research. J Gen Intern Med 2014;29:1692-701.

34 Forsythe LP, Carman KL, Szydlowski V, et al. Patient engagement in research: early findings from the patient-centered outcomes research Institute. Health Aff 2019;38:359-67.

35 Harrison JD, Auerbach AD, Anderson W, et al. Patient stakeholder engagement in research: a narrative review to describe foundational principles and best practice activities. Health Expect 2019;22:307-16.

36 Dodd S, Clarke M, Becker L, et al. A taxonomy has been developed for outcomes in medical research to help improve knowledge discovery. J Clin Epidemiol 2018;96:84-92.

$37 \mathrm{WHO}$. International classification of functioning, disability and health (ICF): World health organisation 2017

38 What matters most to patients with thyroid dysfunction in Malaysia: a preliminary qualitative analysis. endo 2021. USA. The Endocrine Society 2021.
39 Gargon E, Williamson PR, Young B. Improving core outcome set development: qualitative interviews with developers provided pointers to inform guidance. J Clin Epidemiol 2017;86:140-52.

40 Chevance A, Tran V-T, Ravaud P. Controversy and debate series on core outcome sets. paper 1: improving the generalizability and credibility of core outcome sets (COS) by a large and international participation of diverse stakeholders. J Clin Epidemiol 2020;125:206-12.

41 McMillan SS, King M, Tully MP. How to use the nominal group and Delphi techniques. Int J Clin Pharm 2016;38:655-62.

42 DelphiManager [program]. Liverpool, UK: comet initiative 2018.

43 Murphy MK, Black NA, Lamping DL, et al. Consensus development methods, and their use in clinical Guideline development. Health Technol Assess 1998;2:1-88.

44 Guyatt GH, Oxman AD, Kunz R, et al. Grade guidelines: 2. framing the question and deciding on important outcomes. J Clin Epidemiol 2011;64:395-400.

45 Schmitt J, Kottner J, Lange T. Controversy and Debate Series on Core Outcome Sets. Paper 6: Improving the generalizability, credibility and implementation of core outcome sets - the example of the Cochrane Skin-Core Outcome Set Initiative (CS-COUSIN). J Clin Epidemiol 2020;125:229-31.

46 Gorst SL, Prinsen CAC, Salcher-Konrad M, et al. Methods used in the selection of instruments for outcomes included in core outcome sets have improved since the publication of the COSMIN/COMET guideline. J Clin Epidemiol 2020;125:64-75.

47 Mokkink LB, Terwee CB, Patrick DL, et al. The COSMIN checklist for assessing the methodological quality of studies on measurement properties of health status measurement instruments: an international Delphi study. Qual Life Res 2010;19:539-49.

48 Kirkham JJ, Clarke M, Williamson PR. A methodological approach for assessing the uptake of core outcome sets using ClinicalTrials.gov: findings from a review of randomised controlled trials of rheumatoid arthritis. BMJ 2017;357:j2262. 